\title{
DINÁMICAS CAPITALISTAS PARA LA ACUMULACIÓN POR DESPOJO
}

\section{CAPITALIST DYNAMICS FOR THE ACCUMULATION BY DISPOSSESSION}

\author{
Adrián Alejandro Vilchis Onofre* \\ Lilia Zizumbo Villarreal ${ }^{* *}$ \\ Neptalí Monterroso Salvatierra**** \\ Emilio Gerardo Arriaga Álvarez ${ }^{* * * *}$ \\ Alejandro Palafox Muñoz ${ }^{* * * * *}$
}

RESUMEN

\begin{abstract}
El capitalismo se ha valido históricamente de transformaciones para salir de las crisis inmanentes a su lógica, lo cual ha requerido refuncionalizar elementos de modo que le permitan expandirse. La naturaleza es clave en este proceso de maximizar ganancias y encontrar nuevas formas de acumulación, convirtiéndola en una mercancía. En este artículo se analiza el proceso que da origen y reproduce dichas formas de acumulación. El abordaje se da a través de la propuesta de tres dinámicas: penetración, subsunción y cercamientos. Mediante estas dinámicas se muestra la capacidad del sistema capitalista de adaptarse y expandirse para la obtención de ganancias incluso en época de crisis.
\end{abstract}

PALABRAS CLAVE: CONSERVACIÓN DE RECURSOS * CAPITALISMO * TURISMO * RECURSOS NATURALES * CIENCIAS AMBIENTALES

\footnotetext{
* Universidad Autónoma del Estado de México. adrianvilchis@yahoo.com

** Facultad de Turismo y Gastronomía de la Universidad Autónoma del Estado de México. lzv04@yahoo.com

*** Facultad de Ciencias Políticas y Sociales de la Universidad Autónoma del Estado de México. neptali.monterrososalvatierra@gmail.com

**** Instituto de Estudios Sobre la Universidad Autónoma del Estado de México. egearriaga@hotmail.com

***** Universidad de Quintana Roo. alejandro.palafox.munoz@gmail.com
} 


\section{ABSTRACT}

Capitalism has used historically of transformations to emerge from the crisis immanent to its logic, which has required re use elements that will enable it to expand. Nature is key in this process of maximizing profits and find new forms of accumulation, turning it into a commodity. This article discusses the process that gives rise and reproduces these forms of accumulation. The approach is given through the proposal of three dynamics: penetration, subsumption, and enclosures. Through these dynamics demonstrates the ability of the capitalist system adapt and expand to obtain profits even in times of crisis.

KEYWORDS: CONSERVATION OF RESOURCES * CAPITALISM * TOURISM * NATURAL RESOURCES * ENVIRONMENTAL SCIENCES

\section{INTRODUCCIÓN}

La destrucción de la naturaleza y sus consecuencias para la humanidad, son cada vez más evidentes. Se hacen día a día más visibles en la forma en que los fenómenos naturales impactan en la sociedad, convirtiéndose en desastres, ante los cuales el hombre es vulnerable. Más allá de su vínculo con temas aislados como: contaminación del aire, agua, suelo, desertificación o generación de residuos; el eje común está en las formas de producción, que rompen la relación hombre-naturaleza, destruyendo la relación ancestral con esta, dejando de lado la dependencia con ella para la vida y considerándola exclusivamente materia prima del ciclo económico, lo cual destruye su orden complejo y la desnaturaliza para insertarla en el mercado.

Actualmente, los gobiernos locales e instituciones internacionales impulsan esta desnaturalización del ambiente con más fuerza que nunca, a través de la articulación de políticas, la creación de ideologías y el desarrollo tecnológico, lo cual da lugar al surgimiento de las llamadas nuevas formas de acumulación (Harvey 2007).

Este tipo de acumulación tiene como característica la conjunción de la apropiación con la explotación, que aunque son procesos inmanentes del capitalismo, tiene ciertas particularidades en esta nueva forma, entre estas, que se manifiestan como respuesta ante la crisis para contrarrestar la tendencia decreciente de la tasa de ganancia a la cual tiende el sistema y requiere buscar de manera permanente nuevos horizontes hacia los cuales expandirse, para lo cual inserta en su lógica, actividades, sociedades $y$ bienes que no funcionaban de ese modo. La naturaleza en conjunto con las comunidades, además del desarrollo de nuevas formas de explotación, se vuelven un tema fundamental, que da lugar a la desposesión. Es clave para este proceso, la renovada importancia que se da actualmente a las zonas rurales de los países periféricos, que se refuncionalizan para dejar de ser productores solo de alimentos básicos, diversificándose hacia la siembra de flores y frutas, forestería, generación de servicios ambientales e incluso, en sitios recreativos y turísticos.

Dentro de esta lógica, todo (incluso la naturaleza) debe servir para algo y tiene que ser útil a la producción de capital para ser reconocido como real, transformando la vida en un mundo más de medios que de fines, desechando todo lo que se vincule con algún fin último (Horkheimer 2010).

En los últimos 30 años se ha evidenciado esta desposesión a través de una serie de políticas, que se encargan esencialmente de privatizar bienes naturales: mercantilizando agua, tierra, medios de comunicación, transporte, banca, seguridad social, educación, empleo e incluso, la cultura (fiestas patronales, ferias $y$ conocimiento tradicional).

En este artículo se busca analizar el proceso que da origen y reproduce la acumulación por desposesión. Será abordado a través de la propuesta de tres dinámicas: penetración, subsunción y cercamientos.

La primera consiste en una fase de transformaciones estructurales, que son establecidas por los gobiernos en conjunto con organismos 
internacionales, a través de la creación de instituciones y políticas, sentando así las bases legales y normativas para dar paso a nuevas actividades económicas, que permitan flexibilizar la acumulación.

En la segunda dinámica, se aplican las reformas estructurales establecidas previamente, para lo cual se imponen formas violentas de control y dominación en las comunidades, mediante el Estado y Organizaciones No Gubernamentales (onG), haciéndolos partícipes de las nuevas actividades y evitando de este modo la resistencia.

La última dinámica se refiere a la articulación de las dos anteriores: una vez que se han modificado las reglamentaciones e integrado a las comunidades que eran dueñas hasta entonces de los recursos, convirtiéndolas en sujetos dóciles, se hace posible la entrada del capital privado, convirtiendo la naturaleza en mercancía y permitiendo la reproducción del sistema.

Este análisis tiene por objeto señalar que las dinámicas históricas del capitalismo para la acumulación, se continúan manifestando en las "nuevas" formas de acumulación, en el proceso de mercantilización de la naturaleza. Es decir, la base del capitalismo sigue siendo la producción de mercancías, con la particularidad de que actualmente, no son exclusivas las que produce de forma directa el hombre, sino la extracción de materiales de la naturaleza y el uso del espacio (territorio y construcción sociocultural), como atractivo estético mediante su refuncionalización. Esto siempre inscrito en un flexible y parcial marco de legalidad, que proporcionan los Estados.

Al hablar de estas dinámicas se ha elegido un orden y clasificación con fines de facilitar su análisis, sin embargo cabe mencionar que no se trata de un proceso acabado o que se presenta de manera lineal, en la realidad se llevan a cabo de modo simultáneo o en distinto orden, de acuerdo con las necesidades del sistema económico.

Cada una de las dinámicas está atravesada por un proceso violento, no necesariamente referido a golpes, sino a la modificación radical de la forma de vida comunitaria, mediante la imposición de formas restrictivas en la relación hombre-naturaleza, para así disciplinar y controlar a los sujetos.

Se da prioridad y profundidad a la acumulación por desposesión y su vínculo con la naturaleza ${ }^{1}$, sin embargo, se considera que no es posible estudiarla dejando de lado la explotación. Si bien este tipo de acumulación no es nuevo, es actualmente cuando se revela con toda claridad.

\section{LA NATURALEZA COMO EJE DE ACUMULACIÓN}

Históricamente, la explotación de la naturaleza por el capital ha sido un tema relevante en el análisis crítico, respecto a lo cual existen distintas referencias (Horkheimer 2010; Grossman 2004 y Heilbroner 2003). Una crítica recurrente al marxismo es que deja de lado la conservación de la naturaleza al centrarse en la producción de riqueza dada por el trabajo humano, sin embargo, este genera valor de cambio, mientras que es la naturaleza la generadora de valor de uso, que es la "riqueza real":

...el trabajo es, en primer lugar, un proceso entre el hombre y la naturaleza, un proceso en que el hombre media, regula $y$ controla su metabolismo con la naturaleza. El hombre se enfrenta a la materia natural misma como un poder natural. Pone en movimiento las fuerzas naturales que pertenecen a su corporeidad, brazos y piernas, cabeza y manos, a fin de apoderarse de los materiales de la naturaleza bajo una forma útil para su propia vida. Al operar por medio de ese movimiento sobre la naturaleza exterior a él y transformarla, transforma a la vez su propia naturaleza. Desarrolla las potencias que dormitaban en ella y sujeta a su señorío el juego de fuerzas de la misma (Marx y Engels 1988, 215).

$1 \quad$ Este artículo conforma el apartado teórico de una investigación más amplia que tiene por objetivo el análisis de las Áreas Naturales Protegidas como instrumento de desposesión de bienes naturales. 
Marx postuló la teoría de rompimiento del metabolismo entre las sociedades humanas y la naturaleza derivada del productivismo capitalista, denominándola: "escisión de la naturaleza” (Löwy 2011; Clark y Bellamy 2012).

La explicación de este rompimiento se da a través de una analogía respecto a la pérdida de nutrientes del suelo (nitrógeno, fósforo y potasio), debido a la exportación de comida y fibras a las ciudades. En vez de ser devueltos a la tierra, se transportaban para su uso en las urbes, quitando así la capacidad de subsistir del suelo.

La producción capitalista... no solo destruye la salud física de los obreros urbanos y la vida espiritual de los trabajadores rurales, sino que también perturba la circulación material entre el hombre y la tierra, $y$ la condición natural eterna de la fertilidad durable del suelo, volviendo cada vez más difícil la restitución al suelo de los ingredientes que le son quitados y que son usados en forma de alimentos, de ropa, etc. ...además cada progreso de la agricultura capitalista no solo es un progreso en el arte de explotar al trabajador, sino en el arte de despojar el suelo; cada progreso en el arte de incrementar su fertilidad por un tiempo, es un progreso en la ruina de las fuentes durables de fertilidad ...La producción capitalista no desarrolla entonces la técnica y la combinación del proceso de producción social, más que socavando al mismo tiempo las dos fuentes de donde mana toda riqueza: la tierra y el trabajador (Marx, citado en Löwy 2011, 67-68).

De esta manera se vincula la explotación humana con la de la naturaleza, basada en la lógica capitalista de la generación inmediata de ganancias, despilfarrando la fuerza del suelo, que se contrapone al tratamiento conscientemente racional de la tierra como se da en las comunidades rurales.

\section{ACUMULACIÓN POR DESPOSESIÓN}

Recientemente se ha retomado el análisis del papel de la naturaleza en el capitalismo a partir de la noción de acumulación por desposesión (Harvey 2007), que explica la manera en que el sistema se apropia de espacios — que estaban hasta ese momento fuera del mercado-, para expandir sus alcances.

La acumulación por desposesión proviene del análisis de Harvey (2007) de la acumulación originaria de Marx y fue acuñado para enfatizar las nuevas formas que tiene este proceso en la época actual. De Angelis (2012, 1) menciona que "la acumulación originaria no puede ser reducida a un acontecimiento histórico pasado", sino que es parte inherente y continua de los sistemas capitalistas maduros. La acumulación originaria es la imposición del rompimiento de las condiciones de vida de las personas, a través de la separación violenta de estas con sus medios de producción. Actualmente al referirse a la acumulación por desposesión, no solo se trata de la separación del hombre con los medios de producción, sino de los elementos que le son necesarios para la vida, es decir, se rompe el vínculo con la naturaleza. Esta acumulación se hace más evidente que nunca, al mostrarse abiertamente un repentino interés de incluir a las zonas rurales, a las comunidades $y$ a toda actividad ajena hasta entonces a la lógica del sistema, en el proceso de generación de capital (Merchand 2013).

Durante la acumulación originaria, el siervo dejó de ser del señor feudal, para convertirse en un hombre libre, al cual lo único que le pertenecía era su fuerza de trabajo, la cual se vio obligado a vender; en cambio, en la acumulación por desposesión, el hombre posee además de su fuerza de trabajo, un territorio, que le fue otorgado por el mismo Estado que ahora pretende quitárselo.

Esto es claro en el medio rural, donde a los campesinos se les otorgaron tierras, de las cuales depende su subsistencia material y espiritual. Sitios a los que se les confinó, con funciones específicas de producción de alimentos para las zonas urbanas, de acuerdo con el modelo de desarrollo histórico. Sin embargo, ante las crisis en la generación de ganancias del capitalismo, se hace necesario buscar formas de expansión, que subsanen las pérdidas, para lo cual se echa mano de estos sitios, hasta ahora 
relegados, es decir, se basa en el cercamiento de los bienes comunes, que se convirtieron en obstáculo para la consolidación de la clase capitalista (Galaffasi 2009), y se busca nuevas formas de valorizarlos, explotando de manera simultánea hombre y naturaleza.

Al respecto Holloway (Composto y Navarro 2012) menciona que es necesario ver la desposesión y la explotación, como un proceso que es parte de la totalidad del sistema y no de manera aislada, ya que dividir el análisis y dejar de lado alguna, limita la comprensión de que no se puede dar la reproducción sin la subsunción simultánea de ambos.

Esto dentro de una serie de condiciones violentas, pero legales, en donde el papel del Estado es fundamental, puesto que lejos de la idea que sostienen algunos teóricos del neoliberalismo (Hardt y Negri 2000), este no adelgaza ni desaparece, sino que se difumina su actuación al quedar sus funciones repartidas en otros actores, que aparecen como neutrales como las ong $\mathrm{u}$ organismos internacionales, pero que en la mayoría de los casos, se encuentran respaldadas por el poder de un país hegemónico.

La acumulación por desposesión surge en etapas emergentes del capitalismo, cuando la reproducción ampliada peligra por la resistencia social $y$ se fortalece como un medio de expansión hacia nuevos territorios con el fin de reconfigurar las condiciones de acumulación. En otras palabras, la acumulación originaria opera como una estrategia reactiva del capital mientras el avance de la lucha de clases quedaba al margen.

Harvey (2004) menciona que los procesos de desposesión son constitutivos e intrínsecos a la lógica de la acumulación del capital y representan la contracara de la reproducción ampliada. La desposesión se refiere a procesos predatorios, que surgen en momentos de crisis de la reproducción ampliada, como una forma de ajuste $y$ de obtención de ganancias en momentos en que el capital financiero es inestable. Esto se logra a través de la incorporación de "nuevos territorios, ámbitos, relaciones sociales y/o mercados futuros que permitan su realización rentable" (Harvey en Merchand 2013, 114). No es posible la existencia de una acumulación sin la otra, puesto que se dan tanto de forma paralela, sosteniéndose mutuamente, como mediante la preeminencia de una sobre la otra.

La desposesión, de acuerdo con Merchand (2013), se da a través de distintas estrategias como son:

a) Privatización y mercantilización: transferencia de activos públicos o populares a dominio privado, $y$ privilegios de clase.

b) Financiarización: la desregulación permitió al sistema financiero, convertirse en uno de los principales centros de actividad redistributiva, a través de la especulación, la depreciación y el fraude.

c) Gestión y manipulación de la crisis: la creación, la gestión y la manipulación de la crisis a escala mundial ha evolucionado hacia la redistribución deliberada de la riqueza desde los países pobres hacia los ricos.

d) Redistribuciones estatales: el Estado, una vez neoliberalizado, se convierte en el primer agente en la aplicación de las medidas redistributivas, invirtiendo el flujo de la riqueza, desde las clases altas hacia las clases más bajas.

Una diferencia entre los anteriores y los actuales procesos de desposesión, es el marco de legalidad en que se dan, ya que el primero implicaba un quebranto de la ley y una visible violencia corporal o física, mientras que los más recientes actúan bajo una serie de normativas, creadas por el Estado para legitimar el actuar del sistema capitalista. Así, mientras en los primeros se trataba de fundar la propiedad privada como pilar fundamental de la sociedad, en la actualidad se trata de expandir los espacios alcanzados por la propiedad privada o recuperar aquellos ámbitos en los cuales había tenido que ceder cierto terreno (Galafassi 2009).

Cabe destacar que el papel del Estado es fundamental para el desarrollo de dichos procesos, el cual está presente en cada una de las dinámicas de acumulación de distintas formas, pero siempre sosteniendo los intereses del capitalismo. 
El Estado se presenta como una organización jerarquizada de instituciones, reglas, leyes y personal. Dividida en un conjunto visible de dependencias (ejecutivo, legislativo y judicial), además de una red invisible de relaciones de poder, dominio y fuerza que atraviesan a la sociedad. Estas tienen la capacidad de crecer o disminuir en diversos momentos, acorde con la necesidad de reproducción del capital y/o de los acuerdos políticos de las clases dominantes.

Igualmente puede modificarse su jerarquía, permitiendo que la condensación de poder político se desplace en el interior del aparato estatal (Osorio 2009); es decir, se trata de un espacio flexible a las necesidades del sistema económico.

\section{DINÁMICAS DE PENETRACIÓN: FASE DE TRANSFORMACIONES ESTRUCTURALES}

Las modificaciones que se realizan en esta etapa son de tipo estructural, es decir, se modifica de manera sustancial el marco político, normativo e institucional referente a las nuevas actividades económicas o formas de desarrollo que se pretende generar.

Estas nuevas actividades no surgen de manera espontánea o autónoma por parte de cada Estado, sino que son impulsadas desde los países hegemónicos, para imponer el modelo de uso $y$ aprovechamiento de recursos naturales que convienen al capital. Algunos organismos internacionales como el Fondo Monetario Internacional (FMI) y el Banco Mundial (BM) ${ }^{2}$, cumplen la función de ocultar la relación de dominación entre el primer y tercer mundo, dando un aspecto de supuesta equidad, dentro de un mundo globalizado, donde se dice que las contradicciones que evidenciaba el imperialismo, han quedado superadas (Petras y Veltmeyer 2006). De esta manera se pretende romper las barreras políticas e institucionales que impiden la reproducción del capital.

2 Estas instituciones se presentan como independientes a cualquier Estado, pretendiendo una postura neutral, sin embargo se encuentran bajo control de las potencias hegemónicas: Estados Unidos y la Unión Europea, que son quienes determinan sus políticas y representantes.
El comienzo de esta dinámica se hizo evidente alrededor del año 1982, con la sustitución del modelo keynesiano por el neoliberalismo, momento en el cual el papel del Estado transitó de ocuparse de la política social y económica, a la liberalización total, impulsando de este modo la incorporación al mercado de cualquier recurso, tanto humano como natural.

El gobierno se convirtió en "Estado nacional de competencia" (Hirsch en Basualdo y Arceo 2006), encargándose de generar las bases requeridas para todo el proceso de acumulación e interviniendo para realizar los ajustes necesarios cuando el sistema entre en crisis.

La base de este proceso fue el cambio en el orden mundial del capitalismo, a través de la generación de nuevos valores.

Los países periféricos dejaron de ser importantes por sus espacios rurales como productores de granos, lo cual se convirtió en prioridad para los países centrales, de ese modo se tomó control no solo del territorio, sino de la alimentación a escala global, reconfigurando así el sistema en su totalidad.

La utilidad de los países periféricos y sus zonas naturales, fue la posibilidad de incorporarlas de manera eficiente al sistema productivo, para dejar de ser subaprovechadas a través de la agricultura, de esta forma se puso la mira en el agua, en la riqueza mineral y en la diversidad biológica, tanto de flora como de fauna existente en dichos países, los cuales son claves para los procesos de reproducción del capital.

Para su explotación es necesaria la modificación de las formas tradicionales de propiedad, para dar paso a la privatización, sin embargo para facilitar la transición se requirió hacerlo mediante el Estado, el cual teniendo como trasfondo acuerdos internacionales como el Consenso de Washington, cambió la política social y económica, dejando a los campesinos sin apoyo, como hombres libres y propietarios de porciones de tierra que debían trabajar para su reproducción, pero que al cambiar las condiciones que sustentaban su actividad, se vieron forzados a buscar alternativas, entre las que se estaban impulsando por el mismo Estado.

De este modo surgió la "nueva ruralidad", como una alternativa de desarrollo para 
este sector. De acuerdo con la nueva ruralidad, los campesinos deben revalorizar sus bienes naturales y buscar distintas formas de explotarlos, mediante actividades como la forestería, elaboración de artesanías, siembra de frutos y de productos para agrocombustibles, además del turismo, todo bajo el discurso de evitar la destrucción del ambiente.

En esta fase el principal objetivo, es sentar las bases para la introducción al mercado tanto de los bienes comunes, como de los servicios y derechos cívicos.

\section{DINÁMICA DE SUBSUNCIÓN. FASE DE ESTABLECIMIENTO DE CONDICIONES}

Al hablar de subsunción ${ }^{3}$ se hace referencia a la forma en que el capital toma control sobre los recursos naturales para su reproducción, desapareciendo por completo su valor de uso y exaltando el valor de cambio. Esta etapa se caracteriza por ser instrumentada en conjunto entre el Estado y la sociedad civil, a diferencia de la primera en que el actor central era el Estado, en esta fase no puede llevar las acciones a cabo por sí solo.

Para el desarrollo, se crea una ideología que legitima las modificaciones estructurales previas y que asegure el cumplimiento de estas.

El principal instrumento en la actualidad es la sustentabilidad, un concepto ampliamente difundido como apellido de una gran cantidad de actividades, desde agricultura hasta industria, pero que se utiliza en contextos tan variados que pierde capacidad explicativa, además de estar cargado de una ideología acorde con el sistema dominante.

Bajo esta premisa de conservación se crean condiciones para el proceso de desposesión. Uno de los principales mecanismos es el cambio de propiedad del territorio. En esta fase

$3 \quad$ El término "subsunción" traduce el sustantivo "subsumtion" (de origen latino, pero que existe como término técnico en alemán e inglés) que significa tanto "subordinación" como "inclusión". Se refiere a la forma en que el capital subordina (somete) al trabajo, incluyéndolo en su propio concepto, tejiendo entre ambos una "relación interna" (Pagura 2008). lo que originalmente era de una comunidad, pasa a control estatal y se reubica a las poblaciones (que ya han sido convencidas de las bondades de estos nuevos proyectos) o restringe el uso de determinados recursos; esto se evidencia en instrumentos como las Áreas Naturales Protegidas (ANP).

Las ANP son una estrategia que se utiliza para desregular y regular los recursos, impulsando disímiles formas de explotación. De este modo, se da un proceso que comienza con el paso de propiedad comunitaria a estatal, donde se crean las condiciones adecuadas para la privatización, lo cual es el fin último. Todo en un marco de legalidad, pues al convertirse en ANP, la administración corresponde al Estado o se añaden a la tutela de acuerdos internacionales como Ramsar ${ }^{4}$, para el caso de los manglares, que funcionan bajo el auspicio del FMI y BM, así como de corporaciones multinacionales.

Otra función de las ANP es que se instalan como laboratorios in situ para la investigación de propiedades medicinales de plantas y animales, con la finalidad ser patentados ${ }^{5}$ tras su modificación genética. Al ubicarse en comunidades donde ya se tengan conocimientos al respecto, se ahorra dinero y tiempo, intercambiando infraestructura por conocimientos tradicionales, cuyas ganancias se van a empresas privadas, por lo que las comunidades no obtienen mayores beneficios que empleos, los cuales no transforman de manera significativa sus condiciones de vida.

$4 \quad$ El acuerdo Ramsar entró en vigor en 1975 y es el único tratado global relativo al medio ambiente que se ocupa de un tipo de ecosistema en particular. Sus acciones están enfocadas a la cooperación internacional en pro de la conservación de los manglares, para lo cual selecciona los sitios prioritarios en cada país y financia operaciones de restauración y/o preservación. Cada país miembro aporta recursos financieros para su mantenimiento, además recibe apoyo de organismos internacionales como el Banco Mundial y empresas como Danone.

5 Cabe resaltar que la idea de patente encubre una forma de privatización de los recursos, en la cual las comunidades rurales, pierden el derecho a usar libremente la flora $y$ fauna por considerarse propiedad de empresas transnacionales, tras su modificación genética. 
El papel del estado es fundamental en esta etapa, ya que anteriormente ha creado las condiciones políticas y legales necesarias que eliminan restricciones de acceso y uso de los recursos, de manera que promuevan y faciliten su mercantilización. Los acuerdos internacionales determinan las políticas que han de impulsarse $y$ por tanto, los recursos que se consideran estratégicos en determinado momento y los cuales es necesario liberar para agilizar su explotación.

El proceso de subsunción de la naturaleza genera resistencia en los pueblos afectados, ante lo cual es necesario generar estrategias que permitan el control de las manifestaciones en contra.

En este sentido, el Estado utiliza formas violentas de control, no necesariamente referidas a golpes físicos, sino de una coerción casi mecánica de los cuerpos: "movimientos, gestos, actitudes, rapidez; poder infinitesimal sobre el cuerpo activo". En palabras de Foucault, se trata de una anatomía política, que define "cómo se puede apresar el cuerpo de los demás, no simplemente para que ellos hagan lo que se desea, sino para que operen como se quiere, con las técnicas, según la rapidez y la eficacia que se les determina" (Foucault 2009, 159-160).

Por un lado, esto se da en forma dual, se aumenta la fuerza del cuerpo para que sea económicamente útil, mientras por el otro lado, se disminuye la misma, para que sean políticamente obedientes.

La puesta en marcha de estas técnicas disciplinarias no son nuevas, sino que siempre han servido para responder a exigencias coyunturales (Foucault 2009).

Un instrumento más de esta dinámica es la creación y fortalecimiento de Organizaciones de la Sociedad Civil (osc), que remplazan la función que anteriormente estaba a cargo del Estado en cuanto al apoyo social. A través de estas se muestra preocupación tanto por el ambiente como por la sociedad, de modo que son un medio para entrar en las comunidades que se pretenden integrar en el sistema $y$ con el fin de explotarlas y despojarlas, todo bajo la dirección $y$ auspicio de los países dominantes.

Durante esta dinámica, el Estado pierde visibilidad en comparación con la primera, puesto que algunas de sus funciones pasan a manos de las osc. El Estado se encarga de la construcción de carreteras e infraestructura en general, para la llegada de inversiones, además acaba con las estructuras sociales tradicionales, así como, el debilitamiento y desaparición de sindicatos que puedan resistirse y poner en riesgo las nuevas actividades.

Como parte de las formas de control y disciplina de esta etapa, se pone en marcha el cumplimiento de las leyes de la primera dinámica, imponiendo sanciones ejemplares a quienes no cumplan con lo establecido, señalándolos como detractores del progreso.

\section{DINÁMICA DE CERCAMIENTOS: FASE DE DESARROLLO DE LA NUEVA FORMA DE ACUMULACIÓN}

Esta dinámica concluye el ciclo de desposesión, ya que es en ella en la que se hace visible la puesta en marcha de las nuevas actividades que fueron preparadas durante las fases anteriores.

Los recursos naturales tras haber pasado a control estatal, se ofertan como una mercancía a la inversión y pasan a su control a través de concesiones, las cuales son legales (por las modificaciones en cuanto a políticas), pero representan una forma de privatización.

El uso y los beneficios que se obtienen de los recursos dejan de ser para las comunidades originalmente propietarias, las cuales al no ser capaces de cumplir con las reglamentaciones de los nuevos cercamientos de sus bienes, se les ofrece recibir ayuda mediante créditos que ponen en riesgo su patrimonio, o incorporarse como mano de obra en las nuevas empresas, convirtiéndose así en trabajadores de lo que fue suyo.

De este modo, las poblaciones se integran como asalariados en agronegocios, en el turismo o en la minería, entre otras actividades, de lo contrario, se verían forzados a migrar, puesto que no tienen más alternativas para subsistir. Son contratados por medio de outsourcing (empresas reclutadoras de empleados), las cuales no ofrecen prestaciones sociales y se trabaja por bajos salarios.

En esta etapa, el capital privado crea la estructura necesaria para la realización de sus actividades, lo cual se lleva a cabo con la entrada 
al mercado de los bienes comunes, concretando así la desposesión.

La acumulación por desposesión que se practica en América Latina es la que se aplica al modelo conocido como extractivismo, bajo la cual se obtienen grandes cantidades de recursos naturales sin procesar, para ser exportados, industrializados $y$ posteriormente, devueltos a los países en forma de productos de un costo más alto del inicial y revalorizados. Esta explotación de la naturaleza se concesiona a empresas trasnacionales, que tienen base en todo el mundo, bajo el esquema de renta de tierra, en el que transfieren las ganancias a sus países de origen y dejan en los sitios devastación, contaminación y pobreza (Merchand 2013).

Los cercamientos implican entonces el despojar a los hombres (Galafasi 2006), subsumiendo su vida $y$ todo lo que implica al capital, quien tiene supremacía sobre el uso de la naturaleza para darle valor de cambio, significa eliminar la posibilidad de desarrollo de las comunidades, marginándolas a la pobreza al despojarlas no solo de sus medios de producción, sino de los recursos naturales de los cuales dependen física, biológica e incluso, espiritualmente para sobrevivir.

CUADRO 1

PRINCIPALES ASPECTOS DE ANÁLISIS EN CADA UNA DE LAS DINÁMICAS

\begin{tabular}{lcc}
\hline \multicolumn{1}{c}{ DINÁMICA } & CONSISTE EN: & ACTORES VISIBLES \\
\hline \multicolumn{1}{c}{ Dinámica de penetración } & Transformaciones estructurales & Estado \\
\hline - Efectos del cambio de modelo económico (1982) & \\
- Apoyos eliminados & & \\
- Creación de ANP & & \\
- Cambio de propiedad (comunitaria a estatal) & \\
\end{tabular}

\footnotetext{
- Creación de ideología (sustentabilidad)

- Uso del ANP (límites y restricciones)

- Reglamentos

- Formas de coerción y disciplina (incentivos)

- Visibilidad del Estado (participación de ONG)

- Desmantelamiento de organizaciones tradicionales

- Créditos

- Concesiones
}

\begin{tabular}{l} 
Dinámica de cercamientos $\quad \begin{array}{c}\text { Puesta en marcha de las nuevas } \\
\text { actividades }\end{array}$ \\
\hline - Surgimiento de actividades alternativas a la agricultura \\
- Entrada de empresas \\
- Venta de tierras \\
- Agronegocios \\
- Turismo \\
- Forestería
\end{tabular}

Fuente: Elaboración propia.

\section{CONCLUSIONES}

La exposición realizada durante el presente artículo no pretende ser una explicación acabada, sino delinear las estrategias mediante las cuales el capitalismo se apropia de ciertos elementos de la naturaleza, para su explicación y concretar la acumulación por desposesión. Como se mencionó al inicio, no se trata de una estructura inamovible, sino de dinámicas que permanecen en movimiento $y$ que se configuran de acuerdo al tiempo $y$ espacio en que se desarrollan. 
La forma en que se presenta el capitalismo en la actualidad, muestra particularidades respecto al pasado, principalmente el fortalecimiento de formas de acumulación, que se dan de manera simultánea, generando un proceso mucho más violento que en otros tiempos.

Actualmente todo es susceptible de convertirse en mercancía, dado que las barreras de protección a la sociedad que en otras épocas sostuviera el Estado, han desaparecido, lo que permite la integración incluso de los servicios que otorgaba a las corporaciones multinacionales y por tanto, al mercado (salud, educación, vivienda, desarrollo social, etc.).

El Estado juega un papel fundamental en la desposesión, ya que contrario a la imagen que se trata de mostrar, en que se considera que se contrae y que tiende a la desaparición, al ser vaciado de su poder frente a la influencia ejercida por las corporaciones; este, siguiendo los lineamientos internacionales, crea condiciones de legalidad, que dan un rostro humano a las políticas que se instrumentan, facilitando el proceso de acumulación y perdiendo toda soberanía bajo el control de los países dominantes.

La acumulación por desposesión es una muestra de las formas que tiene el sistema capitalista de adaptarse y expandirse para la obtención de ganancias, incluso en época de crisis. Bajo esta lógica se están dando en la actualidad reformas referentes a otros bienes públicos con el fin de privatizarlos, tal es el caso de la educación, que mediante modificaciones en la ley se busca convertirla en una mercancía, susceptible de ser comercializada por el sector privado.

La producción de mercancías sigue siendo el principal motor del capitalismo, sin embargo, su propia lógica lo ha puesto en una crisis, al colocar los recursos que necesita para su reproducción en situación de escasez. Ante lo cual responde con la búsqueda de nuevos espacios de los cuales apropiarse, no necesariamente referido exclusivamente a ubicaciones geográficas, sino a espacios sociales que no se manejaban con las dinámicas del sistema.

La reconfiguración del capitalismo da nuevos valores $y$ funciones a la naturaleza, de modo que sea susceptible de mercantilizarse $y$ obtener ganancias desde la extracción de recursos, hasta su consumo.

Las dinámicas que se presentaron dan cuenta del proceso que contribuye a la acumulación en la actualidad y que si bien, han sido denominadas "nuevas", son formas que muestran la capacidad de adaptación del sistema ante las crisis. Se encuentran basadas en la generación de mercancías y la explotación del trabajo humano, ahora aunado a evitar el pago por recursos naturales, dando lugar al despojo, en un marco de legalidad dado por el Estado.

Es necesario considerar que aunque estas dinámicas se observan con mayor claridad en la etapa denominada neoliberal, no debe perderse que se trata de formas capitalistas de producción, por lo tanto, se pueden encontrar casi en cualquier momento histórico.

\section{BIBLIOGRAFÍA}

\section{LIBROS}

Basualdo, Eduardo y Enrique Arceo. 2006. "Los cambios de los sectores dominantes en América Latina bajo el neoliberalismo. La problemática propuesta”. Neoliberalismo y sectores dominantes. Tendencias globales y experiencias nacionales, editado por Eduardo Basualdo y Enrique Arceo. Buenos Aires: clacso.

Foucault, Michel. 2003.Vigilar y castigar. México: Siglo XXI.

Grossmann, Henryk. 2004. La ley de la acumulación y del derrumbe del sistema capitalista: una teoría de la crisis. España, Siglo XXI.

Hardt, Michael y Antonio Negri. 2000. Imperio. Massachusetts, Harvard.

Harvey, David. 2007. Breve historia del neoliberalismo. Madrid: Akal.

Harvey, David. 2004. El nuevo imperialismo. Madrid: Akal.

Heilbroner, Robert. 2003. Naturaleza lógica del capitalismo, Madrid, Península.

Horkheimer, Max. 1974. Teoría tradicional y teoría crítica. Buenos Aires, Amorrortu.

Horkheimer, Max. 2010. Crítica de la razón instrumental. Madrid: Trotta. 
Löwy, Michael. 2011. Ecosocialismo. Buenos Aires: El Colectivo.

Marx, Karl, y Federico Engels. 1988. La ideología alemana. Barcelona: Lénia.

Petras, James y Henry Veltmeyer. 2006. Imperio con imperialismo: la dinámica globalizante del capitalismo neoliberal. México: Siglo XXI.

\section{PUBLICACIONES PERIÓDICAS}

De Angelis, Massimo. 2012. "Marx y la acumulación primitiva: el carácter continuo de los 'cercamientos' capitalistas". Theomai 26. Julio-Diciembre.

Clark, Brett, y John Bellamy Foster. 2012. "Imperialismo ecológico y la fractura metabólica global". Theomai.

Composto, Claudia. 2012. "Acumulación por despojo y neoextractivismo en América Latina. Una reflexión crítica acerca del Estado y los movimientos socioambientales en el nuevo siglo". Astrolabio.

Composto, Claudia, y Lorena Navarro. 2012. "El despojo es una estrategia del capital para superar la crisis de la explotación". Theomai.

Galafassi, Guido. 2009. "La predación de la naturaleza y el territorio como acumulación". Herramienta.

Merchand, Marco Antonio. 2013. "El Estado en el proceso de acumulación por desposesión favorece la transnacionalización de la minería de oro y plata en México". Paradigma económico: 107-141.

Osorio, Jaime. 2009. Estado, biopoder, exclusión. Análisis desde la lógica del capital. Barcelona, Anthropos-UAM-X.

Pagura, Nicolás. 2008. "Universidad de Buenos Aires". Cátedras. http://www.catedras. fsoc.uba.ar/heler/16.12.08pagura.htm.

PÁGINAS WEB

Ramsar. 2014. Secretaría española de la convención. http://www.ramsar.org/es

Fecha de ingreso: 24/10/2014 Fecha de aprobación: 25/07/2015 
